\title{
Erratum to: Lymphatic Vessel Invasion and Lymph Node Metastasis in Patients with Clinical Stage I Non-Small Cell Lung Cancer [epub ahead of print]
}

Du-Young Kang ${ }^{1}$ Sungsoo Lee ${ }^{2}$

\footnotetext{
${ }^{1}$ Department of Thoracic and Cardiovascular Surgery, NHIS Ilsan Hospital, Ilsan, Republic of Korea

2 Department of Thoracic and Cardiovascular Surgery, Gangnam Severance Hospital, Yonsei University College of Medicine, Seoul, Republic of Korea

Thorac Cardiovasc Surg 2014;62:e1.
}

Address for correspondence Sungsoo Lee, Department of Thoracic and Cardiovascular Surgery, Gangnam Severance Hospital, Yonsei University College of Medicine, 211 Eonju-ro Gangnam-gu, Seoul 135720, Republic of Korea (e-mail: chestlee@yuhs.ac).

ERRATUM

It has been brought to the Publisher's attention that the superscript numbers appearing with author names are not appearing correctly in the above-mentioned article, published eFirst on June 06, 2014. DOI of the original article is DOI: $10.1055 / \mathrm{s}-0034-1381744$. Correct affiliations should be read as presented above. 